\title{
Incidence and Density Relationships of Powdery Mildew on Apple
}

\author{
Xiangming $\mathrm{Xu}$ and Laurence V. Madden
}

First author: Horticulture Research International-East Malling, West Malling, Kent ME19 6BJ, UK; and second author: Department of Plant Pathology, Ohio State University, Wooster 44691. Accepted for publication 30 April 2002.

\begin{abstract}
Xu, X.-M., and Madden, L. V. 2002. Incidence and density relationships of powdery mildew on apple. Phytopathology 92:1005-1014.

The relationships between disease incidence and colony density and between leaf and shoot disease incidences for apple powdery mildew were investigated over four seasons in order to derive a simple relationship for predicting density using incidence. The Neyman type A distribution generally provided a good fit to the observed number of colonies per leaf and shoot, and provided a significantly better fit than the Poisson distribution, indicating a degree of aggregation of mildew colonies. In general, Taylor's power-law satisfactorily described the observed variancemean relationship for colony density; however, Taylor's power-law broke

tribution for colony density. Regression models using the complementary log-log transformation of incidence also provided accurate predictions of leaf (or shoot) disease incidence from colonies per leaf (or per shoot). Similar accuracies of these incidence-density models suggested that variance-mean ratio of colony density was more or less constant over time. Unlike the case with colony density, the number of mildewed leaves per shoot generally had a random pattern, as indicated by the good fit of the binomial distribution. Thus, it was possible to estimate the leaf incidence of the youngest unrolled leaves on a shoot using the shoot incidence. It is argued that the leaf incidence-density relationships developed in the present study may be used in making practical disease management decisions.
\end{abstract} down at very high levels of mean density. Incidence of leaf infection could be determined based on average number of colonies per leaf assuming a fixed variance-mean relationship and a Neyman type A dis-
Additional keywords: beta-binomial distribution, binary power law, Podosphaera leucotricha.
Accurate disease assessment is usually time consuming and expensive. For a rational disease management program in commercial agriculture, a method for fast, easy, accurate, and reproducible assessment of disease level is important. Usually, disease intensity is measured as both incidence and severity. Disease incidence refers to the number or proportion of plant units that are visibly diseased and can be measured on different scales such as individual leaves, individual shoots, or individual plants (21). Disease severity is the area or volume of plant tissue that is diseased relative to the total area or volume; sometimes disease severity is measured as the number of colonies (or lesions) per plant unit (disease density) (5). In general, incidence is easier and quicker to assess than severity. However, severity may be a more important and useful measurement for many diseases that arise in research and practical disease management. Similarly, incidence at a higher scale (e.g., plants) is easier and quicker to assess than incidence at a lower scale (e.g., leaves and shoots), although incidence of the lower scale (i.e., at the primary scale in the hierarchy) may be of greater interpretive value (16).

Much research has been conducted to study relationships between disease incidence and severity measures, and between disease incidence assessed using plant units at different scales. These relationships, if proved accurate and reliable, can be used to estimate severity or incidence at low scales from easily recorded incidence data at high scales, which would be very useful, especially for commercial disease management, because of time constraints in disease assessment. Incidence-severity relationships have usually been studied using correlation and regression analysis (27). More mechanistic models of this relationship can be

Corresponding author: X.-M. Xu; E-mail address: xiangming.xu@hri.ac.uk

Publication no. P-2002-0717-01R

(C) 2002 The American Phytopathological Society derived if certain assumptions are made regarding the frequency distribution of density data (5). Incidence-severity relationships have been investigated for several pathosystems, including coffee rust $(26,29)$, powdery mildew and leaf rust of wheat (17), banana leaf spot (6), Paspalum leaf blight (25), and apple cedar rust (10).

Recently, considerable research effort has been directed at establishing functional relationships between disease incidences at two levels in a spatial hierarchy, based on the binomial and betabinomial distributions (16,31). If the incidence is binomially distributed (i.e., random), incidence at the lower level can be predicted from the incidence at the higher level using a formula based on the binomial distribution. However, disease incidence data often are not fitted well by the binomial distribution; the betabinomial distribution has often been more suitable for representing diseased individuals because of aggregation of disease at some spatial scale $(14,15,21)$. The beta-binomial distribution cannot be easily used to predict incidence of the lower scale from observations at the higher scale, although an approximate method has been developed based on the degree of aggregation of disease at the lower scale (22).

Field assessment of secondary powdery mildew (caused by Podosphaera leucotricha) on apple is generally difficult. In contrast to the distinct colonies that occur in the greenhouse, secondary mildew in the field typically occurs as an expansive and diffuse colony on the abaxial leaf surface, which may be confused with leaf hairs on some cultivars. Several studies have been conducted to relate disease incidence to disease density (number of mildew colonies per leaf) for apple powdery mildew $(3,28)$. Incidence of leaf infection is proportional to the square root of disease density; this relationship does not vary between cultivars or locations but does vary with season (28). Butt and Barlow (3) predicted density from the leaf incidence data assuming that colony density per leaf follows a negative binomial distribution and using Taylor's power law to relate mean colony density to the aggrega- 
tion parameter of the negative binomial distribution for colony density. In the same study, the incidence of shoot infection (only including the top five youngest fully unrolled leaves) was a good predictor of the incidence of leaf infection when leaf infection incidence was less than $15 \%$, based on the assumption of binomial distribution of infected leaves. For higher levels of leaf incidence, the prediction based on shoot incidence (and randomness of infected leaves) was greater than the observed, indicating some aggregation of infected leaves on a shoot (16). There appear to be no differences between cultivars in this shoot-leaf incidence relationship (3). However, it is not known whether the density-incidence relationship varies between cultivars, locations, or seasons. For a density-incidence relationship to be used for practical disease assessment, it is essential that such dependence on cultivar, location, or season be minimal.

The goal of this study was to further explore the relationships between incidence and density at the same scale and across scales (leaf and shoot) with the overall aim of finding a simple and robust relationship for predicting disease density using either leaf or shoot incidence. To achieve this overall goal, we needed to (i) characterize the dynamics of the heterogeneity of disease density at each scale and disease incidence at the leaf scale; (ii) characterize the disease incidence relationship between the two scales; and (iii) develop models for accurately predicting disease density from incidence at the same scale or across scales, based on fairly empirical regression models, statistical distributions, or other measures of heterogeneity. The first and second specific objectives were required to fully achieve the third objective, because a proper understanding of disease heterogeneity and spatial scaling will lead to more robust prediction models $(16,27,33)$. Secondary mildew was assessed frequently and nondestructively over four seasons to develop prediction models.

\section{MATERIALS AND METHODS}

Disease assessment. From 1994 to 1997, two neighboring orchards of apple cv. Cox on M9 rootstock (planted in 1989), with Malus pollinators, were used for disease assessment. Secondary mildew was assessed frequently and nondestructively (usually three times each week, depending on the weather) from late May/early June to late July/early August.

At the beginning of each season, 10 trees were selected systematically in each orchard and four vegetative shoots were chosen at random on each selected tree, two on each side of the alley way. On each recording date, leaf 0 was tagged with a label; leaf 0 is the youngest unrolled leaf on an extension shoot. Leaves at position $-1,-2$, and -3 , etc., are older and increasingly larger than leaf 0 , leaf -1 being adjacent to leaf 0 . The numbers of visible colonies on leaves at positions $-1,-2,-3$, and -4 were counted. Leaf 0 was not assessed because its small size and often very hairy surface in orchard conditions made assessment impractical. The leaves sampled are those most important for practical disease management, because most infectious colonies are on these leaves (18). In total, approximately 320 leaves were assessed on each date. All the assessments were made on the top four leaves of the same shoots within each season. On each recording date, if the number of colonies was smaller than the previous time on the

TABLE 1. Some of properties of three two-parameter distributions fitted in this study ${ }^{\mathrm{a}}$

\begin{tabular}{llll}
\hline Properties & $\begin{array}{l}\text { Negative } \\
\text { binomial }\end{array}$ & Polya-Aeppli & Neyman type A \\
\hline Overall mean & $\lambda$ & $\lambda /(1-\tau)$ & $\lambda_{1} \lambda_{2}$ \\
Variance & $\lambda(1+\lambda / k)$ & $\lambda(1+\tau) /(1-\tau)^{2}$ & $\lambda_{1} \lambda_{2}\left(1+\lambda_{2}\right)$ \\
$P(0)$ & $(1+\lambda / k)^{-k}$ & $\exp (-\lambda)$ & $\exp \left\{\lambda_{1}\left[\exp \left(-\lambda_{2}\right)-1\right]\right\}$ \\
\hline
\end{tabular}

a $\lambda, k, \tau, \lambda_{1}$, and $\lambda_{2}$ are parameters. same leaf, due to colony merging, the previous assessment was carried over. From the raw data on colonies, the number of diseased leaves (with one or more colonies) and shoots (with one or more diseased leaves) could be determined.

Statistical analysis. Understanding heterogeneity in colony density and incidence, and how heterogeneity changes with disease level (or with time), is of great importance for developing more mechanistic and reliable incidence-density and incidenceincidence models, which may be easier to interpret than empirical regression models $(5,16,22,27)$. In this study, we first determined whether the number of colonies on a leaf or a shoot was randomly distributed by fitting several discrete distributions to the observed data for each individual assessment. Next, Taylor's power-law (30) relationship between variance and mean was used to describe the observed dynamics of heterogeneity in colony density. We exploited several models relating disease incidence to density based on the results from the distribution and power law analyses and also based on empirical curve fitting (23). Finally, we investigated whether it was possible to relate incidences at the two levels (leaf and shoot). This was done to determine whether incidence of shoot infection could be related to incidence of leaf infection, and thus (ultimately) to colony density on leaves. By quantifying distribution and heterogeneity of disease, the robustness of the developed models for predicting either colony density or disease incidence can be considered. Use of these models for other data sets will require that the variance-mean relationship and distribution results hold. All statistical analyses, other than fitting of the beta-binomial distribution, were done using Genstat (Numerical Algorithms Group Ltd., Oxford, England).

Distribution of disease density. The Genstat DISTRIBUTION directive was used to fit observed numbers of colonies (i.e., disease density) on a leaf at each assessment to four commonly used discrete probability distributions: Poisson, negative binomial, PolyaAeppli, and Neyman type A. The Poisson distribution is appropriate when the number of colonies on a leaf is randomly distributed. The other three are two-parameter generalized Poisson distributions $(9,24)$; some of their important properties are listed in Table 1. These can be used to describe overdispersed or aggregated (clustered) data. Parameters were estimated using maximum likelihood and optimized using an iterative Gauss-Newton method. Sampling units in the statistical analysis consists of the individual leaves at each sampling time. The number of frequency classes (groups) was determined automatically by the DISTRIBUTION directive. Goodness of fit was determined with a $\chi^{2}$ test based on the deviance, which is the sum of squared so-called deviance residuals. Distributions were also fitted to all the data sets within each year, or over the 4 years jointly, by assuming a constant variance-mean ratio (VMR). Similar analysis was also applied to the number of colonies per shoot, i.e., shoots were treated as sampling units with all colonies from four assessed leaves per shoot calculated.

Variance-mean relationship of disease density. Taylor's power law (30) was used to describe the relationship between the sample mean $(m)$ and variance $(v)$ of disease density on all the assessed leaves at a single assessment date over the 4 years

$$
V=a m^{b}
$$

When $a=b=1$, there is no aggregation or overdispersion over the full range of means. For parameter estimation, a linearized form of equation 1 was used, i.e.,

$$
\ln (v)=\ln (a)+b \ln (m)
$$

Differences in the two parameters between the 4 years were tested. This analysis was done for both numbers of colonies on a leaf and on a shoot.

Relating incidence to density. In all the analyses for incidence in relation to density, there were 78 data points, i.e., the mean 
number of colonies on a leaf (or shoot) and the incidence of leaf (or shoot) disease at each individual assessment over the 4 years.

Based on Poisson distributions. An incidence-density relationship was derived by assuming that the number of colonies on a leaf followed a Poisson distribution. Then, the probability of a leaf being infected $(p)$, which is estimated by the proportion of diseased leaves, is related to the mean number of colonies on a leaf by the equation

$$
p=1-P(0)=1-\exp (-m)
$$

where $m$ is, as before, the average number of colonies on a leaf, and $P(0)$ is the theoretical probability of leaves with zero colonies. Thus, given an estimate of $p, m$ can be estimated by rearrangement of equation 3 as $-\ln (1-p)$.

Based on Neyman type A distributions and a constant VMR. Preliminary results from fitting the four distributions to observed data indicated that numbers of colonies on a leaf was best described by a Neyman type A distribution (described below). Thus, the incidence of leaf infection was related to the two distribution parameters $\left(\lambda_{1}, \lambda_{2}\right)$ (Table 1$)$ by the equation

$$
p=1-P(0)=1-\exp \left\{\lambda_{1}\left[\exp \left(-\lambda_{2}\right)-1\right]\right\}
$$

Furthermore, for Neyman type A distributions, the mean colony number $(m)$ is $\lambda_{1} \lambda_{2}$. Thus, assuming a constant $\lambda_{2}$ over assessments (i.e., constant VMR of $1+\lambda_{2}$; Table 1), equation 4 becomes

$$
p=1-P(0)=1-\exp \left\{\left(m / \lambda_{2}\right)\left[\exp \left(-\lambda_{2}\right)-1\right]\right\}
$$

This was, essentially, a regression problem, i.e., estimating parameter $\lambda_{2}$ from the observed data ( $p$ and $m$ ) with a constraint based on an assumed property of heterogeneity (fixed VMR). The Genstat FITNONLINEAR directive was used to fit equation 5 to the observed data. Thus, given an estimate of $p, m$ can be estimated by rearrangement of equation 5 using the fitted parameter $\lambda_{2}$ value as

$$
\left.m=\lambda_{2} \ln (1-p) / \exp \left(-\lambda_{2}\right)-1\right]
$$

Based on Neyman type A distributions and power-law relationships. It is not possible to estimate the mean number of colonies $\left(\lambda_{1} \lambda_{2}\right)$ directly from $p$ using equation 4 because there are two unknowns $\left(\lambda_{1}, \lambda_{2}\right)$. However, assuming a common variance-tomean relationship of equation 1 for heterogeneity across all data sets, one can substitute $\lambda_{1} \lambda_{2}\left(1+\lambda_{2}\right)$ for $v$ and $\lambda_{1} \lambda_{2}$ for $m$. Solving for $\lambda_{1}$ leads to

$$
\lambda_{1}=\frac{1}{\lambda_{2}}\left(\frac{1+\lambda_{2}}{a}\right)^{\frac{1}{b-1}}
$$

where $a$ and $b$ are parameters of equation 1. By substituting equation 6 for $\lambda_{1}$ in equation 4 , it is possible now to use $p$ to estimate the average number of colonies on a leaf. This is done by first solving equation 4 for $\lambda_{2}$ and then determining $m$ as

$$
\lambda_{1} \lambda_{2}=\left(\frac{1+\lambda_{2}}{a}\right)^{\frac{1}{b-1}}
$$

using equation 6 . However, there is no explicit solution for $\lambda_{2}$ in terms of $p$, and only iterative solutions are possible. When $b=1$, equation 1 can be written as $\lambda_{1} \lambda_{2}\left(1+\lambda_{2}\right)=a \lambda_{1} \lambda_{2}$. Thus, $\lambda_{2}=a-1$ and $\lambda_{1}=m /(a-1)$. This leads to the same equation as equation 5, with $\lambda_{2}$ given by $a-1$. With $b=1$, a constant VMR is specified, with $\mathrm{VMR}=a$.

Empirical regression analysis. The complementary log-log transformation of the disease incidence, $\operatorname{CLL}(p)=\ln [-\ln (1-p)]$, was directly regressed on the logarithmic transformation of the average number of colonies on a leaf, $\ln (m)$ :

$$
\operatorname{CLL}(p)=\ln (\gamma)+\delta \ln (m)
$$

which corresponds to the relationship $p=1-\exp \left(-\gamma m^{\delta}\right)$. This represents a two-parameter generalization of the Poisson model (23); the Poisson model is a special case with $\gamma=1, \delta=1$. The inverse of equation 7, often referred to as the Kono-Sugino equation, has been widely used in entomology to estimate mean pest population density from the incidence of infestation (19).

Distribution type and regression analyses were conducted to relate shoot incidence to numbers of colonies over the four leaves on a shoot (i.e., colonies per shoot).

Relating incidences at two levels. If the frequency distribution of the number of diseased leaves on a shoot follows a binomial distribution (i.e., a random pattern of diseased leaves over shoots), the proportion of shoots with at least one diseased leaf (i.e., incidence of shoot infection: $p_{\text {shoot }}$ ) is given by

$$
p_{\text {shoot }}=1-\left(1-p_{\text {leaf }}\right)^{n}
$$

where $p_{\text {leaf }}$ is the incidence of leaf infection and $n$ is the number of assessed leaves per shoot (four in the current study). For other nonrandom patterns of diseased leaves on a shoot, such as those giving rise to the beta-binomial distribution, an approximate relationship between $p_{\text {shoot }}$ and $p_{\text {leaf }}$ has been derived (22). The binomial distribution was first fitted to individual data sets of leaf incidence on a shoot. Beta-binomial distributions were only fitted to those data sets that showed a significant deviation from the binomial distribution based on a $\chi^{2}$ goodness-of-fit test. BBD software was used for fitting binomial and beta-binomial distributions (20).

A binary form of power law $(13,21)$ was used to investigate the temporal dynamics of aggregation of diseased leaves on a shoot. It related the observed variance $(V)$ of leaf disease incidence between shoots to the variance expected $\left(V_{R}\right)$ if a binomial distribution is assumed for the number of diseased leaves per shoot:

$$
V=A V_{R}^{\beta}=A[p(1-p) / n]^{\beta}
$$

in which $A$ and $\beta$ are parameters. At each sampling time, the variance $(V)$ of disease incidence between shoots was calculated. For parameter estimation, a linearized form of equation 9 was used,

$$
\ln (V)=\ln (A)+\beta \ln [p(1-p) / n]
$$

When $A=\beta=1$, there is no aggregation or overdispersion.

\section{RESULTS}

General epidemic development in the $\mathbf{4}$ years. The observed mildew epidemics in the 4 years are shown in Figure 1, which shows the average numbers of colonies on a leaf and the incidence of leaf infection on each assessment date. There were large differences in the disease density and in the patterns of disease development over time between the 4 years. The observed density and incidence were generally low, except in 1997. Disease incidence in 1997 was already very high $(\approx 58 \%)$ on the first assessment date.

Distribution of disease density. In all the years except 1997, fewer than $50 \%$ of the leaves had mildew colonies when data were pooled over all the assessment dates within 1 year (Fig. 2). Most data sets of numbers of colonies on a leaf or on a shoot could not be adequately described by the Poisson distribution (Table 2). Only 9 and 20 out of a total of 78 assessments could be described satisfactorily by a Poisson distribution for leaf and shoot 
density data, respectively. Of those data sets that could not be adequately fitted by a Poisson distribution, most were satisfactorily fitted by any of the two-parameter distributions (Table 2). Out of 60 data sets fitted to the two-parameter distributions, 54 showed significantly better fit than the Poisson distribution $(P<$ $0.05)$ based on the differences in deviances. Of the three distributions, the Neyman type A appeared to fit the data best for leaf density data. Only six data sets showed significant deviations from the fitted Neyman type A distributions compared with 15 and 14 for negative binomial and Polya-Aeppli distributions, respectively. The six data sets that failed to fit a Neyman type A distribution also failed to fit negative binomial and Polya-Aeppli distributions. However, it is not possible to test statistically which of the three distributions is most appropriate because they are not nested.

For shoot density data, almost all the data sets were well fitted by the three tested two-parameter distributions, and not fitted by the Poisson distribution (Table 2). Again, for most data sets, the two-parameter distributions fitted the data significantly better than the Poisson based on differences in deviances.
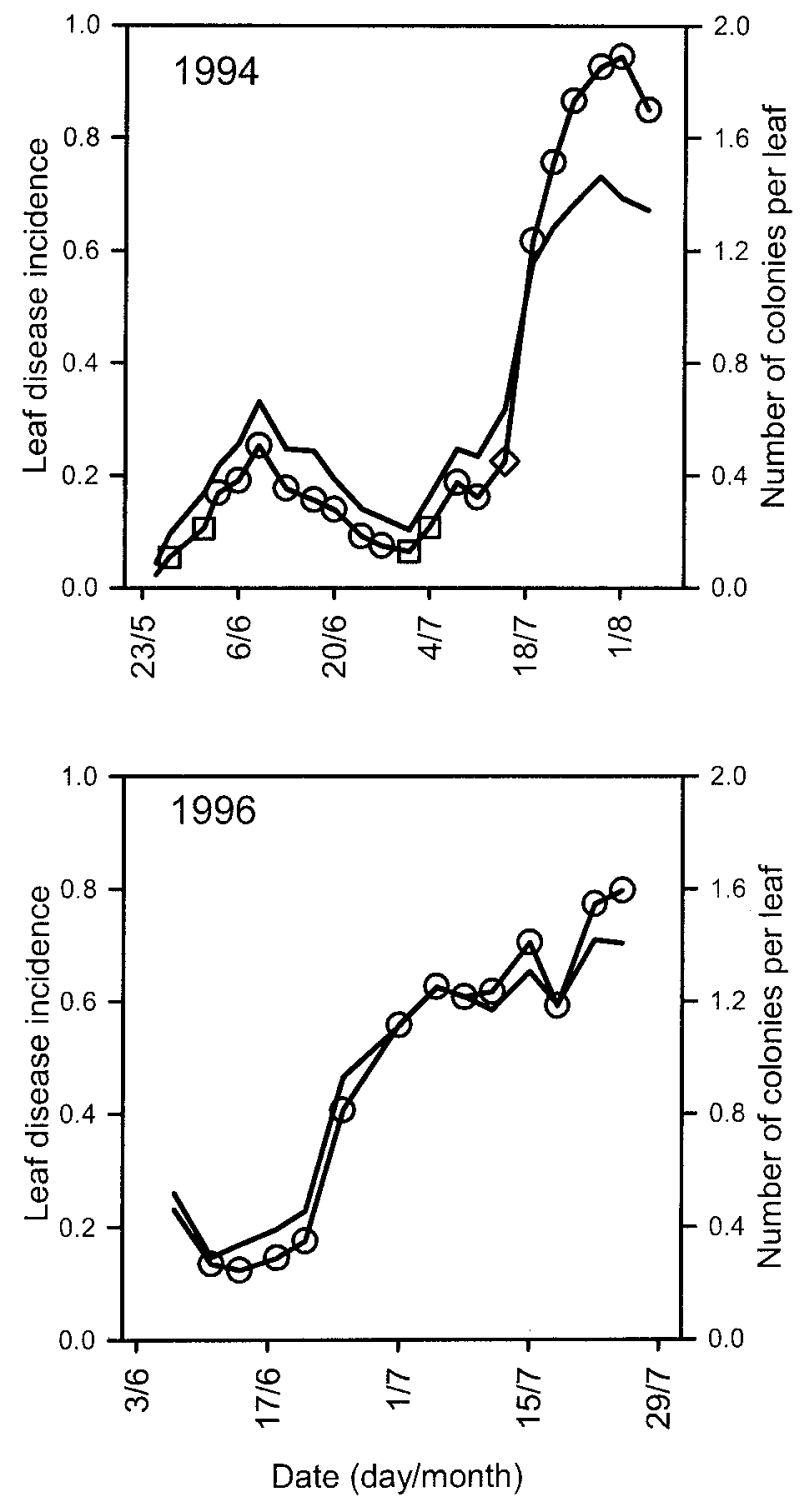

Variation in $\lambda_{1}$ of the Neyman type A distribution between assessments within seasons was much greater than variation in $\lambda_{2}$, especially in 1997. $\lambda_{1}$ ranged from 0.27 to 4.23 , with an average of 1.58 for 1994 to 1996 ; in 1997, it ranged from -25.95 to 82.64 , with an average of 4.33 . $\lambda_{2}$ ranged from -0.24 to 1.01 , with an average of 0.58 for 1994 to 1996; in 1997, it ranged from -0.27 to 0.70 , with an average of 0.09 . There was no significant correlation between $\lambda_{1}$ and $\lambda_{2}$. When fitted with a common $\lambda_{2}$ (i.e., common VMR $\left[1+\lambda_{2}\right]$ ) within each year, there were significant differences in the goodness of the fit compared with the individual fit at $5 \%$ significance level for 1994 and 1996, and at 1\% significance level for 1997, indicating heterogeneity of $\lambda_{2}$ between assessments. The maximum likelihood routine failed to fit Neyman type A distributions with a common $\lambda_{2}$ for data over all 4 years because of nonconvergence. Analysis of variance showed that there were no significant differences in $\lambda_{1}$ between the 4 years and that $\lambda_{2}$ was significantly smaller in 1997 than in the other years. Similar results were obtained for numbers of colonies per shoot.

Variance-mean relationship of disease density. In general, $\ln (v)$ increased linearly with increasing $\ln (m)$ for numbers of col-
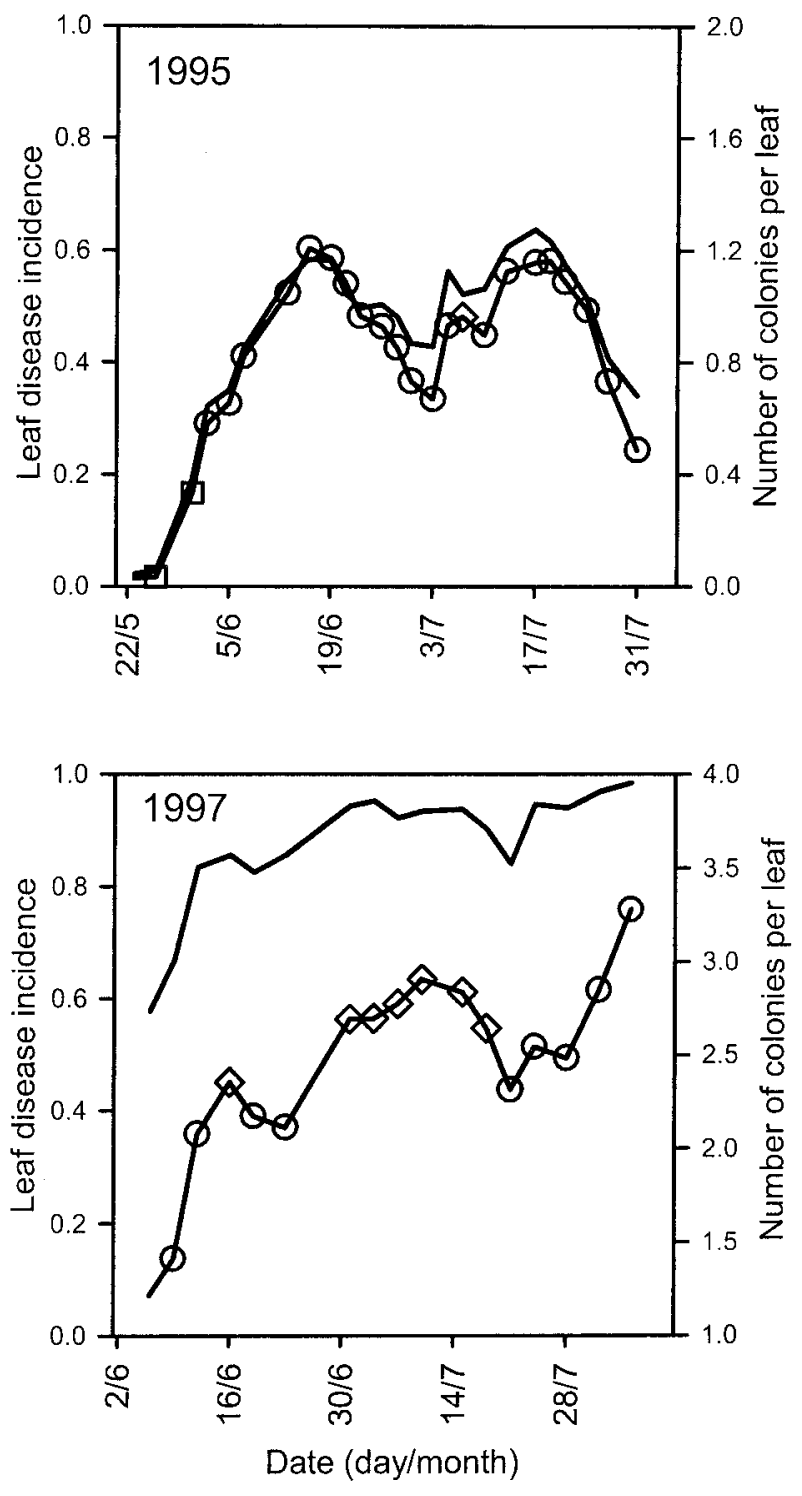

Fig. 1. Observed dynamics (average number of colonies on a leaf and incidence of leaf infection on each assessment date) of apple powdery mildew in 1994 to 1997. Square $=$ assessment for which degree of freedom is zero or optimization is not possible when fitted to a Poisson distribution; circle $=$ assessment for which there are significant deviations from fitted Poisson distributions; diamond = assessment for which there are no significant deviations from fitted Poisson distributions; line with symbols $=$ average number of colonies on a leaf; and line without symbols $=$ leaf incidence. 
onies on a leaf and on a shoot (Fig. 3). Most assessments in 1997 were clustered in a small top-right region of the graph and the variance-mean relationship was very weak, with $\ln (v)$ decreasing slightly with increasing $\ln (m)$. Most of the variation in the variance-mean relationship between the 4 years was due to the difference between 1997 and the other 3 years. Although there were significant differences in the slope parameter ( $b$ in equation 2 ) among the 3 years (1994 to 1996), these accounted for only an additional $1 \%$ of the total variation in $\ln (v)$. Thus, a common regression model was fitted for 1994 to 1996 :

$$
\ln (v)=0.435+1.022 \ln (m)
$$

(0.029) (0.026)

and the standard errors of parameter estimates are in the brackets below the corresponding parameter. This equation accounted for approximately $96 \%$ of total observed variation in $\ln (v)$. The estimate of $a$ in equation 1 is $\exp (0.435)=1.545$. The parameter $a$ was significantly greater than $1(\ln (a)>0)$. The estimate of the slope parameter $b$ was not significantly different from $1(P>$ $0.05)$, but significantly greater than $0(P<0.001)$. Thus, although there were significant changes in $\lambda_{2}$ among assessments (described previously), the VMR $\left(=a=1+\lambda_{2}\right)$ over time was more or less constant, as indicated by $b \approx 1$. That is, because $b \approx 1$, VMR did not systematically increase or decrease with increasing $m$ (or increasing assessment time).

Similar results were obtained for numbers of colonies on a shoot. Again for 1997, the variance-mean relationship was very weak. Although there were significant differences in the slope parameter among 1994 to 1996, these accounted for an additional $3.5 \%$ of the total variation in $\ln (v)$. Thus, a common regression equation was fitted for 1994 to 1996 :

$$
\ln (v)=0.699+1.079 \ln (m)
$$

(0.058) (0.046)

which accounted for $90 \%$ of the total variation in $\ln (v)$. Estimated $a$ was $\exp (0.699)=2.012$, significantly greater than $1(\ln (a)>0)$. The estimate of the slope parameter was not significantly different from $1(P>0.05)$, but significantly greater than $0(P<0.001)$,

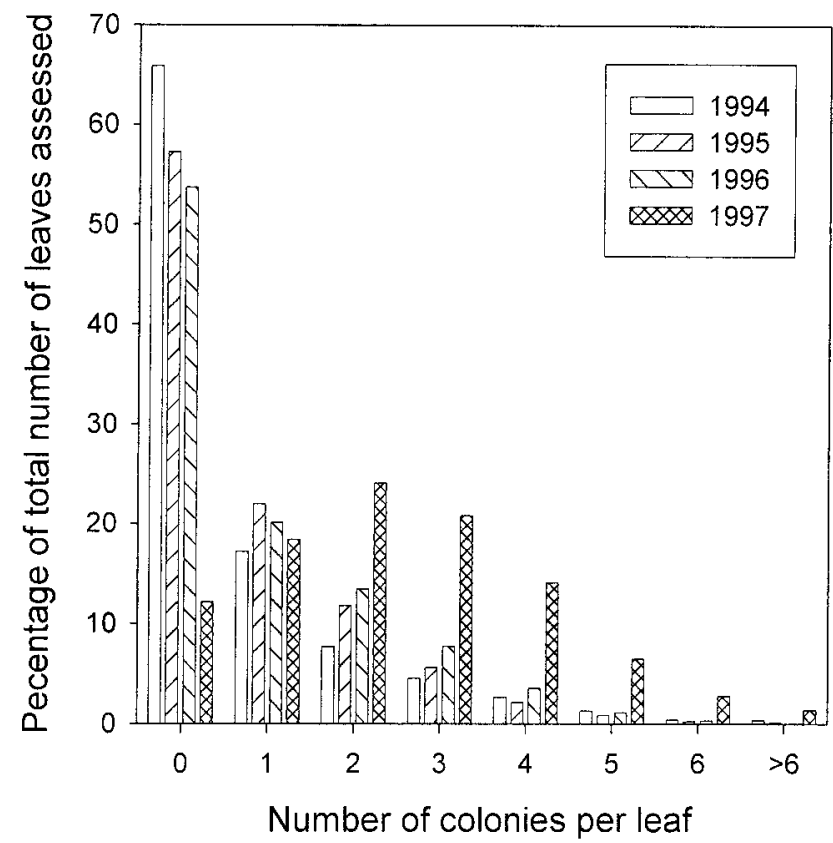

Fig. 2. Frequency distributions of numbers of mildew colonies on a leaf pooled over all the assessments in the 4 years. thus again indicating an aggregated pattern but with a constant VMR over time.

Relating incidence to density. In all the analyses relating incidence to density, data from 1997 were excluded because of the results of variance-mean analysis and other practical considerations discussed later. Thus, all the fitting results applied only to 1994 to 1996 data, although the data and predicted values for 1997 are shown in the graphs.

Leaf incidence. When the number of colonies on a leaf was assumed to follow a Poisson distribution, predicted numbers of colonies using leaf incidence (equation 3 ) were generally greater than observed (Fig. 4A) even though approximately $96.2 \%$ of variation in the observed incidence was accounted for by equation 3. This overestimation was due to the aggregated patterns of the number of colonies on a leaf as shown by the distribution analysis.

The relationship between leaf incidence and number of colonies on a leaf was derived by assuming that the number of colonies per leaf followed a Neyman type A distribution and assuming a constant $\lambda_{2}$ (i.e., VMR $\left[1+\lambda_{2}\right]$ ) over all the assessments. The derived model is

$$
p=1-P(0)=1-\exp \{(m / 0.606)[\exp (-0.606)-1]\}
$$

and the standard error of estimated $\lambda_{2}$ is 0.026 . The fitted values were very close to the observed data (Fig. 4A). Approximately $98.4 \%$ of variation in the observed incidence was accounted for by equation 13 .

The relationship was also derived by assuming a common variance-mean relationship (equation 11) and assuming that the number of colonies per leaf followed a Neyman type A distribution. Thus, using equation 6 ,

$$
\lambda_{1}=\frac{1}{\lambda_{2}}\left(\frac{1+\lambda_{2}}{1.545}\right)^{45.45}
$$

We obtained the following relationship for $p$ by substituting equation 14 for $\lambda_{1}$ in equation 4 :

$$
p=1-P(0)=1-\exp \left[\frac{1}{\lambda_{2}}\left(\frac{1+\lambda_{2}}{1.545}\right)^{45.45}\left[\exp \left(-\lambda_{2}\right)-1\right]\right]
$$

By solving equation 15 (iteratively) for $\lambda_{2}$ and the average number of colonies per leaf $(m)$ was determined as $\lambda_{1} \lambda_{2}=\left[\left(1+\lambda_{2}\right) /\right.$ $(1.545)]^{45.45}$ using equation 14. This relationship between $p$ and $m$

\begin{tabular}{|c|c|c|c|c|}
\hline Category & Poisson & $\begin{array}{l}\text { Negative } \\
\text { binomial }\end{array}$ & $\begin{array}{c}\text { Neyman } \\
\text { Type A }\end{array}$ & $\begin{array}{l}\text { Polya- } \\
\text { Aeppl }\end{array}$ \\
\hline $\begin{array}{l}\text { Colonies per leaf } \\
\mathrm{df}=0^{\mathrm{a}}\end{array}$ & 5 & 16 & 16 & 16 \\
\hline Not optimized ${ }^{b}$ & 1 & 2 & 2 & 1 \\
\hline$\chi^{2}$ significant $^{\mathrm{c}}$ & 63 & 15 & 6 & 14 \\
\hline $\begin{array}{l}\text { Colonies per shoo } \\
\mathrm{df}=0^{\mathrm{a}}\end{array}$ & 3 & 6 & 6 & 6 \\
\hline$\chi^{2}$ significant ${ }^{\mathrm{c}}$ & 55 & 3 & 3 & 1 \\
\hline
\end{tabular}
was evaluated numerically and was very close to the observed re-

TABLE 2. Results of fitting four different probability distributions to the 78 data sets of numbers of colonies of apple powdery mildew recorded over 4 years

${ }^{a}$ Number of data sets in which there were not sufficient degrees of freedom $(\mathrm{df}=0)$ due to very low levels of disease.

${ }^{\mathrm{b}}$ Number of data sets in which optimization failed with no clear reasons.

${ }^{\mathrm{c}}$ Number of data sets in which the $\chi^{2}$ goodness-of-fit test was significant at $P=0.05$. Significance means lack of fit; thus, a small number indicates that most data sets were well fitted by the corresponding distribution. 
lationship (Fig. 4A), accounting for approximately $98.5 \%$ of total variation in $p$ based on manual calculations.

The complementary log-log transformation of the incidence of leaf disease incidence was well related to the average number of colonies on a leaf, excluding the 1997 data:

$$
\begin{aligned}
\operatorname{CLL}(p)= & -0.295+0.956 \ln (m) \\
& (0.014)(0.013)
\end{aligned}
$$

This equation provided very accurate estimates of average numbers of colonies on a leaf using $p$ (Fig. 4A), accounting for approximately $98.5 \%$ of total variation in $p$. There were no sig-
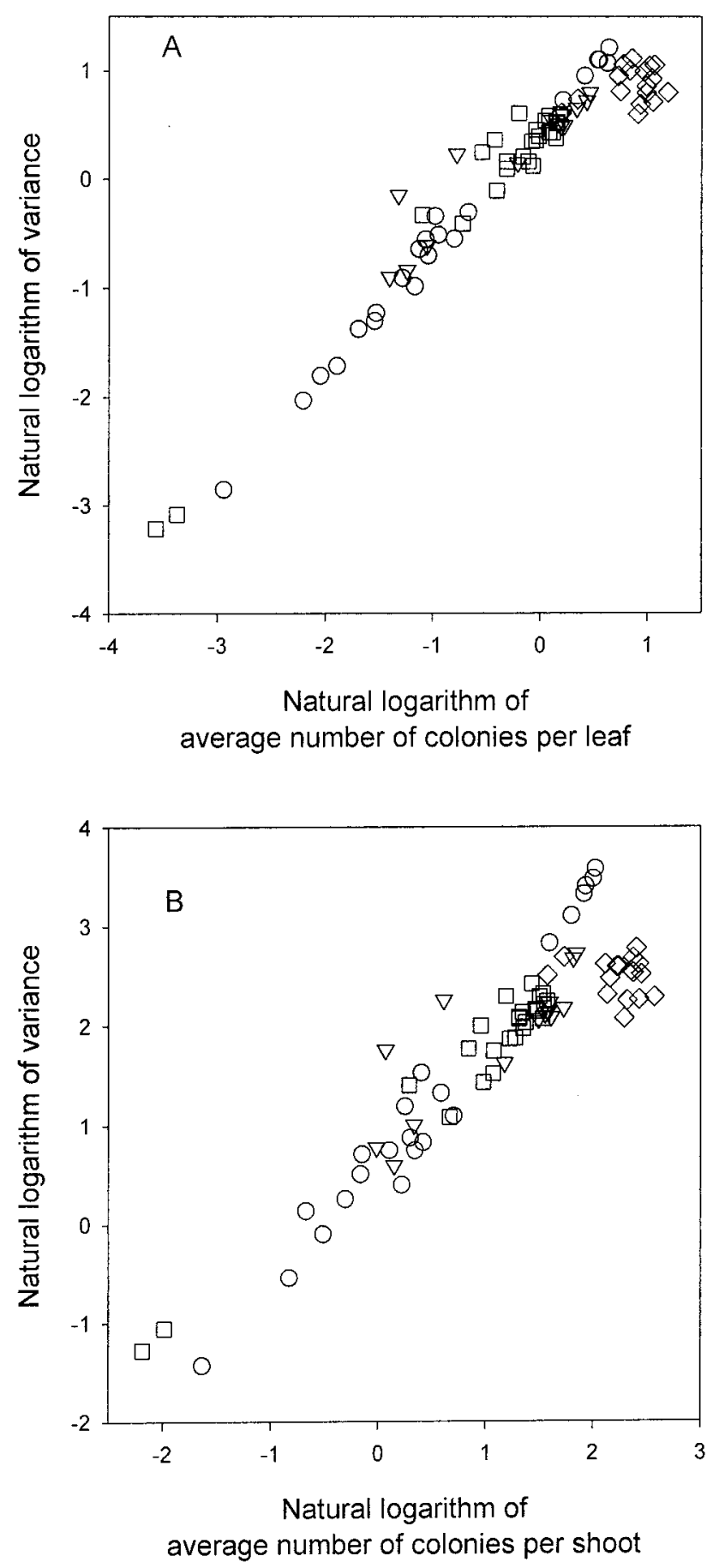

Fig. 3. Observed and fitted power-law relationships between variance and mean of disease density data of apple powdery mildew over the 4 years. Circle $=1994$, square $=1995$, triangle $=1996$, and diamond $=1997$. nificant differences between the 3 years (1994 to 1996) in the two model parameters.

Shoot incidence. Similar results were obtained for the relationship between shoot incidence and average numbers of colonies per shoot based on the distribution results (Fig. 4B). The model based on the Poisson distribution generally overestimated $p$ despite the high percentage (approximately 95.1\%) of total variation in $p$ accounted for by this model. The relationship, based on the assumptions of a Neyman type A distribution and a constant $\lambda_{2}$, is

$$
p=1-P(0)=1-\exp \{(m / 0.892)[\exp (-0.892)-1]\}
$$

and the standard error of estimated $\lambda_{2}$ is 0.066 . Approximately $97.1 \%$ of variation in the observed incidence was accounted for by equation 17.

The relationship was also derived assuming a common powerlaw variance-mean relationship (equation 12) and also assuming that the number of colonies per shoot followed a Neyman type A distribution. Predicted values were very close to the observed relationship (Fig. 4B). This model explained approximately $96.7 \%$ of total variation in $p$ based on manual calculations.

The complementary log-log transformation of the incidence of shoot infection incidence was also well related to the average number of colonies on a shoot:

$$
\begin{aligned}
\operatorname{CLL}(p)= & -0.378+0.959 \ln (m) \\
& (0.0367)(0.0292)
\end{aligned}
$$

This equation provided very accurate estimates of average numbers of colonies on a leaf using $p$ (Fig. 4B), accounting for approximately $97.2 \%$ of total variation in $p$. There were no significant differences between the 3 years (1994 to 1996) in the two model parameters.

Relating incidences on a leaf and on a shoot. The binomial distributions satisfactorily fitted 59 of 78 data sets of leaf disease incidence. Of the 19 data sets that showed significant deviations from fitted binomial distributions, 11 were from 1994 and 16 could be well described by beta-binomial distributions. Estimated values of the aggregation parameter $(\theta)$ of the beta-binomial for these 16 data sets ranged from 0.096 to 0.512 , with a median of 0.265 .

Binary power law satisfactorily fitted the data over the 4 years. The resulting model is

$$
\begin{gathered}
\ln (V)=0.025+0.949 \ln \left(V_{R}\right) \\
(0.130)(0.039)
\end{gathered}
$$

This equation accounted for $88.4 \%$ of total variation in $\ln (V)$. When the year was included as a factor in fitting the model, there were significant differences in the constant and slope parameters between years, but these only accounted for an additional $3 \%$ of the total variation. The estimated constant and slope parameter were not significantly different from 0 and $1(P>0.05)$, respectively, indicating in general that there was no aggregation or overdispersion of infected leaves on a shoot. Assuming a binomial distribution of diseased leaves on a shoot, the incidence of leaf infection could be well predicted from the incidence of shoot infection using equation 8 (Fig. 5). The percentage of total variation in leaf incidence accounted for was approximately $91.3 \%$.

\section{DISCUSSION}

In this study, we explored the relationships between disease incidence and colony density (severity) and between incidences at two levels in a spatial hierarchy of apple powdery mildew, with the aim of predicting disease density using either leaf or shoot 
incidence. In order to relate incidence to disease density using a mechanistic approach, the type of distribution that disease density follows is critical and the incidence can be estimated using the zero term of this probability distribution (16). The present study showed that the number of apple powdery mildew colonies on a leaf generally did not follow a Poisson distribution. This is not surprising given that most plant diseases are not randomly distributed $(16,21)$. Of the three generalized two-parameter distributions considered, the Neyman type A generally provided a good fit to the largest number of data sets, indicating a certain degree of aggregation of mildew colonies on a leaf. This is further confirmed by the variance-mean analysis, which indicated that the variance generally increased linearly with colony density, on a $\log -\log$ scale, as expected based on the overdispersion of individual data sets. Estimated $b$ (equation 1) was close to 1 (equations 11 and 12), however, indicating that the degree of overdispersion (or aggregation) did not depend on the magnitude of the mean. An aggregated pattern of density data is often observed for plant diseases, e.g., tobacco blue mold (32), apple powdery mildew (1), leaf blight of carrot caused by Cercospora carotae (2), apple cedar rust (10), and leek rust (8).

Although the Neyman type A provided a good fit to more data sets than the negative binomial and Poly-Aeppli distributions, differences in the goodness of fit between them (measured by $\chi^{2}$ tests) were generally small. In the literature, disease density data often are explicitly fitted only by the negative binomial distribution. Indeed, a negative binomial model described the number of colonies per leaf of apple powdery mildew satisfactorily (1). There may be several reasons for using negative binomial distributions to describe disease density data. The negative binomial distribution is easier to work with. For example, it is simple to get the zero term of probability class $(P(0))$ in relation to the mean, and thus to incidence. The negative binomial parameter $(k)$ can be incorporated into temporal models to investigate the effect of disease aggregation on temporal disease increase $(32,33)$. Coupling the negative binomial distribution with the variance-mean relationship is also relatively easy (34) and can be used to relate disease density to incidence, allowing for the varying degree of aggregation over time (2). We have demonstrated here, however, that the Neyman type A distribution also can be used effectively to represent colony density of powdery mildew when overdispersion is a power-law function of the mean.

A Neyman type A distribution has two primary derivations (9) either as a generalized (clustered) or compound (mixed) Poisson. The former represents the situation in which there are random initial clusters of colonies (represented by the Poisson), perhaps due to initial infections on a shoot, and random numbers of colonies produced per cluster (also represented by the Poisson). In the context of apple powdery mildew, it should be noted that it takes, on average, 24 days from leaf emergence (position +4$)$ to the last assessment stage (position -4). For cv. Cox, it takes at least 22 days for two generations of colonies, because the average incubation and latent period in field conditions is more than 11 days (4). Therefore, there is insufficient time throughout most of the assessment period to observe more than one generation of colonies, indicating that the generalized Poisson mechanism probably is not the cause of the disease aggregation. For a compound Poisson process, there is a random (Poisson) distribution of colonies at each site (shoot), but the expected or mean value of the distribution varies with the site as a result of heterogeneity in the tree and orchard. If the mean per site has a Poisson distribution, then the Neyman type A is derived. There are many spatial variables that could affect mildew development in field conditions such as inoculum distribution and density, local microclimate, and possibly leaf epiphytes. Therefore, a compound Poisson process is the more likely explanation for the observed aggregation of colonies in this study. It must be emphasized that multiple mechanisms can produce a given statistical distribution (7) so caution should be used when interpreting the causes for a particular pattern.

There were significant differences in the estimates of density distribution parameters over time in 3 of the 4 years based on the significant difference in VMR (i.e., $\lambda_{2}$ of Neyman type A distribution). This is not surprising because disease aggregation characteristics are likely to vary with changes in means $(21,35-37)$. This change in relation to mean is usually modeled by Taylor's power law (30) or its analogue, the binary power law (13). The variancemean relationship (equation 2) generally fitted the observed data
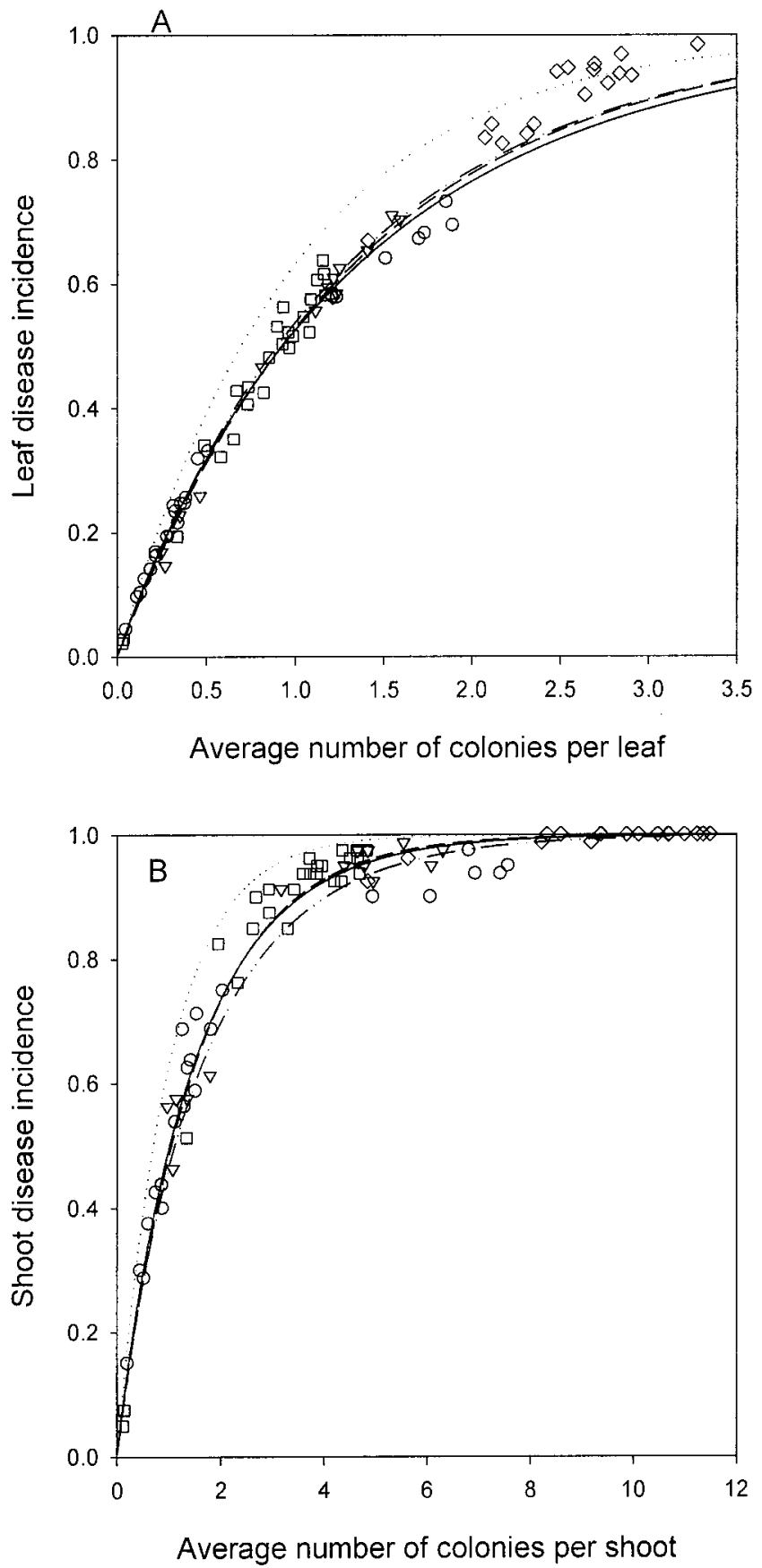

Fig. 4. Observed and fitted relationships between disease density and incidence of apple powdery mildew over the 4 years. Circle $=1994$, square $=$ 1995 , triangle $=1996$, diamond $=1997$, dotted line $=$ fitted curve based on Poisson distributions, solid line $=$ regression model based on complementary $\log -\log$ transformation of disease incidence, dashed line $=$ directly fitted curve based on Neyman type A distributions, and dot-dashed line = indirectly fitted curve based on Neyman type A distributions. The 1997 data were excluded in deriving the relationships. 
well, and differences in the parameter estimates between the 4 years were very small, especially between 1994, 1995, and 1996. For assessments with high average number of colonies per leaf $(>2)$, the variance decreased somewhat with increasing mean numbers of colonies, instead of increasing as expected (all of these assessments occurred in 1997). This apparent decrease in variance with increasing mean is probably due to the limited number of colonies that a leaf could contain. The positive relationship may hold for the whole range of numbers of colonies only if the range of possible numbers of colonies on a leaf is infinite, which is obviously not true. Thus, increasing average number of colonies over a threshold will result in decreases in variance because the differences in the number of colonies between leaves will decrease. This is very similar to the rational for the functional form of the binary power law (13). Indeed, the extreme case would be that the variance is zero but there is the maximum number of colonies on all leaves. For situations like this, the power law may need to be modified to account for the overdispersion in general. On cv. Cox, $100 \%$ disease severity is equivalent to nine colonies per leaf (18). Numbers of colonies observed in the present study were much smaller than those observed by Seem and Gilpatrick in the United States (28), but were consistent with those recorded on field cv. Cox trees in the United Kingdom $(3,18)$.

Four approaches were used to relate disease incidence $(p)$ to density $(m)$. All four approaches are based on the relationship $p=$ $1-\exp [f(m)]$. The methods differ in the precise form of $f(m)$. In method $1, f(m)$ is given simply by $-m$ (i.e., assuming that colonies follow a Poisson distribution). In the other three methods, it is assumed that colony density follows a nonrandom discrete distribution. Prediction accuracy of method 1 was much worse than the others, because colonies are generally aggregated on a leaf or a shoot.

The models derived using methods 2 to 4 showed nearly identical prediction accuracies and differed little between 1994, 1995, and 1996. Equivalence of methods 2 and 3 is due to the fact that the VMR did not change systematically with the mean (or over time) based on the closeness of estimated $b$ (equation 1 ) to 1 .

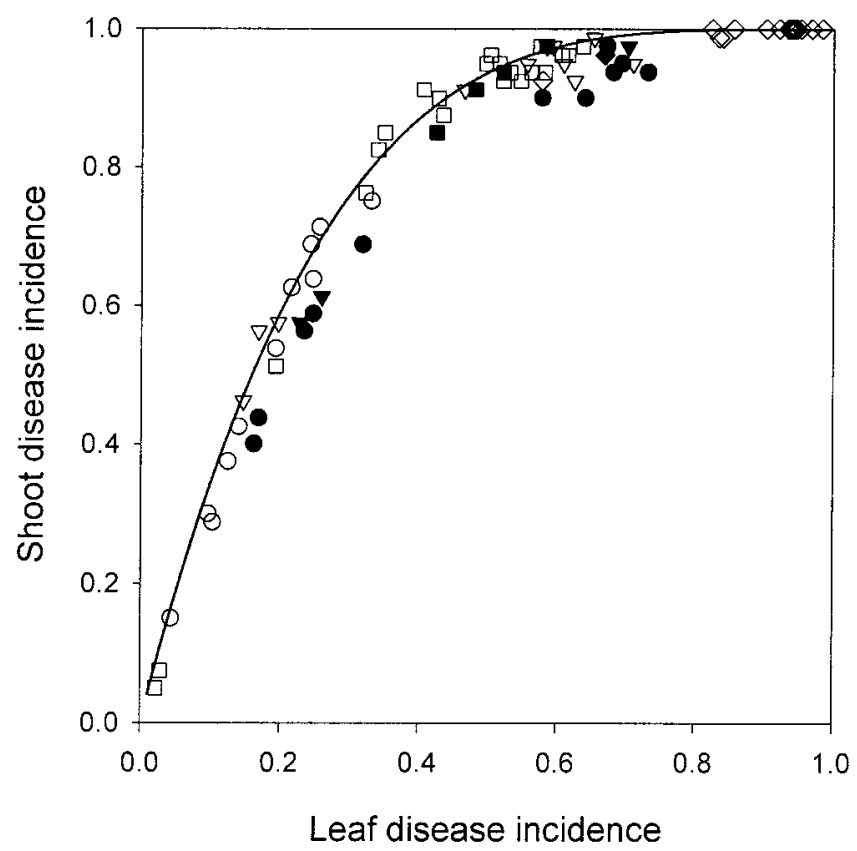

Fig. 5. Observed and fitted relationships between leaf and shoot incidences of apple powdery mildew over the 4 years. Circle $=1994$, square $=1995$, triangle $=1996$, diamond $=1997$, filled symbols $=$ assessments for which the number of diseased leaves showed significant deviations from fitted binomial distributions, and solid line $=$ fitted curve assuming the number of diseased leaves on a shoot is binomially distributed.
Even though the estimated $\lambda_{2}$ of the Neyman type A did vary within a year, the power law results suggest that a constant VMR is a reasonable approximation. In method $2, f(m)=m\left\{\left[\exp \left(-\lambda_{2}\right)-\right.\right.$ $\left.1] / \lambda_{2}\right\}$, where $\lambda_{2}$ is a parameter of Neyman type A distributions for colony density. Thus, in this method, $f(m)$ is simply a linear function of $m$ with an intercept of zero and a slope of $\vartheta=\left[\exp \left(-\lambda_{2}\right)-\right.$ $1] / \lambda_{2}$. Estimates of $\vartheta$ were -0.750 and -0.662 for leaves and shoots, based on the estimated $\lambda_{2}$ values in equations 13 and 17 . Constant $\lambda_{2}$ implies a constant VMR, equal to $1+\lambda_{2}$, estimated here to be $1.606(1+0.606)$ and $1.892(1+0.892)$ for leaf and shoot incidence-density relationships, respectively (equations 13 and 17). In method $3, f(m)=\lambda_{1}\left[\exp \left(-\lambda_{2}\right)-1\right]$, with $m=\lambda_{1} \lambda_{2}$ subject to the constraint that a power-law model describes the variance and mean relationship (equation 1). Assuming $b=1$, then VMR is simply the estimated $a$, equal to 1.545 and 2.012 for leaf and shoot data, respectively, which were very close to the respective VMR values of 1.606 and 1.892 estimated from method 2. Note that there was no curve-fitting of the incidence-density relationship with this method. In method $4, f(m)=-\gamma m^{\delta}$; equivalence of this method with methods 2 and 3 occurs when $\delta$ equals 1 . Indeed, for both leaf and shoot $\operatorname{CLL}(p)$ models, $\delta$ was close to 1 (although significantly different from 1.0 for the leaf model). Estimates of $-\gamma$ were -0.744 and -0.685 for leaf and shoot model, respectively, very close to the corresponding values of $\vartheta(-0.750$ and -0.662 ) of method 2.

The derived incidence-density relationship is not likely to depend greatly on seasons per se but rather on mean number of colonies or overall disease severity, as indicated by the nearly constant VMR. When average colony number is $>2$ (corresponding to an incidence of leaf infection of approximately $75 \%$ ), the incidence-severity relationship will underestimate the leaf disease incidence for the reasons discussed previously. However, this should not cause any problem for using the model in practical disease management, because the threshold for mildew control in the United Kingdom was set to $<8 \%, 8$ to $20 \%$, and $>20 \%$ leaf disease incidence for light, moderate, and severe risks, respectively (3), far less than the $75 \%$ incidence. This is one of the main reasons that the 1997 data were excluded when deriving severityincidence relationships. In a study in the United States, the incidence-severity relationship for apple powdery mildew varied between seasons but remained constant across locations and cultivars (28). The seasonal effect could well be explained by differences in disease severity between seasons. In the Seem and Gilpatrick (28) study, colony density was higher than in our investigation.

In general, the frequency distribution of diseased leaves per shoot was satisfactorily described by a binomial distribution, indicating a random pattern at the spatial scale of the shoots or smaller. Only 19 of 78 data sets showed significant deviations from the fitted binomial distributions; 16 of these data sets could be satisfactorily described by a beta-binomial distribution. This is further confirmed by binary power law analysis, which showed that, overall, there was no significant aggregation or overdispersion over time $(\beta=1)$. Evidently, the 16 overdispersed data sets did not have a large influence on the binary power law results. In this study, however, the statistical power for detecting aggregation of diseased leaves on a shoot might be low because there were only four leaves per shoot (21). Based on the present data, it was possible to estimate the leaf incidence of the youngest four unrolled leaves on a shoot using the shoot incidence based on the binomial distribution (equation 8). Butt and Barlow (3) showed a similar relationship between leaf and shoot incidences for apple powdery mildew. Thus, aggregation in some data sets did not impede using a simple binomial-based model to make predictions. As shown in the Appendix, results for the relationship between colony density and the leaf and shoot disease incidences can be combined to predict the relationship between leaf and shoot incidence for a binomial (random) distribution of incidence. This pre- 
diction is fully consistent with the observed incidence-incidence relationship, justifying the use of leaf disease incidence as the threshold for mildew management (3).

This study shows that there is a degree of aggregation of mildew colonies on leaves and shoots that could be described by the Neyman type A (and other two-parameter distributions), and that the degree of aggregation varies with the mean according to the power law. VMR of colony density is approximately constant between assessments or seasons. However, the incidence of infected leaves was not, in general, aggregated on shoots, as indicated by the binary power law and good fit of the binomial distribution to the data. Thus, it was very feasible to predict both mean colony density and proportion of infected leaves from the proportion of infected shoots (as well as mean colony density from infected leaves). Predictions could be based on pure distributional analysis at the lower scale or on curve fitting of data between scales. Because the relationships were generally consistent over seasons, it should be possible to use them in making efficient disease management decisions based on either incidence or colony density thresholds. However, further validation of these models using independent data is needed.

\section{APPENDIX}

As shown by Hughes and Gottwald $(11,12)$, equation 8 for disease incidence can be written as

$$
\operatorname{CLL}\left(p_{\text {shoot }}\right)=\ln (4)+\operatorname{CLL}\left(p_{\text {leaf }}\right)
$$

when $n=4$. If we use subscripts for the $\gamma$ and $\delta$ parameters of equation 7 when referring to leaves and shoots, we can write

$$
\operatorname{CLL}\left(p_{\text {leaf }}\right)=\ln \left(\gamma_{\text {leaf }}\right)+\delta_{\text {leaf }} \ln \left(m_{\text {leaf }}\right)
$$

and

$$
\operatorname{CLL}\left(p_{\text {shoot }}\right)=\ln \left(\gamma_{\text {shoot }}\right)+\delta_{\text {shoot }} \ln \left(m_{\text {shoot }}\right)
$$

Given that $m_{\text {leaf }}=m_{\text {shoot }} / 4$, we can substitute equation A2 for $\operatorname{CLL}\left(p_{\text {leaf }}\right)$ in equation $\mathrm{A} 1$ and write

$$
\operatorname{CLL}\left(p_{\text {shoot }}\right)=\ln (4)+\ln \left(\gamma_{\text {leaf }}\right)+\delta_{\text {leaf }} \ln \left(m_{\text {shoot }} / 4\right)
$$

which can be rearranged to

$$
\operatorname{CLL}\left(p_{\text {shoot }}\right)=\left(1-\delta_{\text {leaf }}\right) \ln (4)+\ln \left(\gamma_{\text {leaf }}\right)+\delta_{\text {leaf }} \ln \left(m_{\text {shoot }}\right)
$$

If the equations for incidence-density are correct for both shoots and leaves, and the binomial distribution is appropriate for incidence of diseased leaves per shoot, then a direct comparison of equations A3 and A5 for shoot incidence implies that $\delta_{\text {shoot }}=\delta_{\text {leaf }}$ and $\ln \left(\gamma_{\text {shoot }}\right)=\ln \left(\gamma_{\text {leaf }}\right)+\left(1-\delta_{\text {leaf }}\right) \ln (4)$.

The results in equations 16 and 18 show that the numerical results were generally consistent with these theoretical results. For instance, estimates of $\delta_{\text {shoot }}$ and $\delta_{\text {leaf }}$ agreed to two decimal places (0.96), and the direct estimate of $\gamma_{\text {shoot }}(0.68)$ was close to the estimate predicted here $(0.79)$.

\section{ACKNOWLEDGMENTS}

This work was funded by the U.K. Department of Environment, Food and Rural Affairs (DEFRA). We thank J. Robinson for disease assessment, M. Ridout and K. Phelps for comments, and G. Hughes and N. McRoberts for discussing with us some of their research on incidenceseverity and incidence-incidence relationships.

\section{LITERATURE CITED}

1. Barlow, G. P. 1977. Sampling and variation. East Malling Res. Stn. Annu. Rep. 1976:68.

2. Boivin, G., Kushalappa, A. C., and Brodeur, L. 1990. Spatial dispersal and binomial sequential sampling plan for Cercospora carotae on carrots. Can. J. Plant Pathol. 12:209-212.

3. Butt, D. J., and Barlow, G. P. 1979. The management of apple powdery mildew: A disease assessment method for growers. Pages 77-86 in: Proc. Br. Crop Prot. Conf. Pests Dis.

4. Butt, D. J., and Jeger, M. J. 1986. Components of spore production in apple powdery mildew (Podosphaera leucotricha). Plant Pathol. 35:491497.

5. Campbell, C. L., and Madden, L. V. 1990. Introduction to Plant Disease Epidemiology. John Wiley \& Sons, New York.

6. Chung, T. Y., and Jeger, M. J. 1987. Relationship between incidence and severity of banana leaf spot in Taiwan. Phytopathology 77:15371541.

7. Cliff, A., and Ord, J. 1981. Spatial Processes: Models and Applications. Pion Ltd., London, UK.

8. de Jong, P. D. 1995. Sampling for detection: Leek rust as an example. Int. J. Pest Manag. 41:31-35.

9. Freeman, G. H. 1980. Fitting two-parameter discrete distributions to many data sets with one common parameter. J. R. Stat. Soc. Ser. C (Appl. Stat.) 29:259-267.

10. Gregory, P. H. 1948. The multiple-infection transformation. Ann. Appl. Biol. 35:412-417.

11. Hughes, G., and Gottwald, T. R. 1998. Survey methods for assessment of citrus tristeza virus incidence. Phytopathology 88:715-723.

12. Hughes, G., and Gottwald, T. R. 1999. Survey methods for assessment of citrus tristeza virus incidence when Toxoptera citricida is the predominant vector. Phytopathology 89:487-494.

13. Hughes, G., and Madden, L. V. 1992. Aggregation and incidence of disease. Plant Pathol. 41:657-660.

14. Hughes, G., and Madden, L. V. 1993. Using the beta-binomial distribution to describe aggregated patterns of disease incidence. Phytopathology 83:759-763.

15. Hughes, G., and Madden, L. V. 1998. Comment-Using spatial and temporal patterns of Armillaria root disease to formulate management recommendations for Ontario's black spruce (Picea mariana) seed orchards. Can. J. For. Res. 28:154-158.

16. Hughes, G., McRoberts, N., Madden, L. V., and Gottwald, T. R. 1997. Relationships between disease incidence at two levels in a spatial hierarchy. Phytopathology 87:542-550.

17. James, W. C., and Shih, C. S. 1973. Relationships between incidence and severity of powdery mildew and leaf rust on winter wheat. Phytopathology 63:183-187.

18. Jeger, M. J., and Butt, D. J. 1986. Epidemics of apple powdery mildew (Podosphaera leucotricha) in a mixed cultivar orchard. Plant Pathol. 35:498-505.

19. Kono, T., and Sugino, T. 1958. On the estimation of the density of rice stems infested by the rice stem borer. Jpn. J. Appl. Entomol. Zool. 89:770-781.

20. Madden, L. V., and Hughes, G. 1994. BBD-Computer software for fitting the beat-binomial distribution to disease incidence data. Plant Dis. 78:536-540.

21. Madden, L. V., and Hughes, G. 1995. Plant disease incidence: Distributions, heterogeneity, and temporal analysis. Annu. Rev. Phytopathol. 33:529-564.

22. Madden, L. V., and Hughes, G. 1999. An effective sample size for predicting plant disease incidence in a spatial hierarchy. Phytopathology 89:770-781

23. Nachman, G. 1981. A mathematical model of the functional relationship between density and spatial distribution of a population. J. Anim. Ecol. 50:453-460.

24. Ord, J. K. 1972. Families of Frequency Distributions. Griffin, London

25. Price, T. V., and Williams, B. L. 1990. Studies on the severity and incidence of paspalum leaf blight. Aust. J. Agric. Res. 41:377-392.

26. Rayner, R. W. 1961. Measurement of fungicidal effects in field trials. Nature 190:328-330.

27. Seem, R. C. 1984. Disease incidence and severity relationships. Annu. Rev. Phytopathol. 22:137-150.

28. Seem, R. C., and Gilpatrick, J. D. 1980. Incidence and severity relationships of secondary infections of powdery mildew on apple. Phytopathology 70:851-854.

29. Silva-Acuna, R., Maffia, L. A., Zambolim, L., and Berger, R. D. 1999. Incidence-severity relationships in the pathosystem Coffea-Hemeleia vastatrix. Plant Dis. 83:186-188.

30. Taylor, L. R. 1961. Aggregation, variance and the mean. Nature 189: 732-735. 
31. Turechek, W., and Madden, L. 2001. Effect of scale on plant disease incidence and heterogeneity in a spatial hierarchy. Ecol. Model. 144:7795 .

32. Waggoner, P. E. 1981. Models of plant disease. Bioscience 31:315-319.

33. Waggoner, P. E., and Rich, S. 1981. Colony distribution, multiple infection, and the logistic increase of plant disease. Proc. Nat. Acad. Sci. USA 78:3292-3295.

34. Wilson, L. T., and Room, P. M. 1983. Clumping patterns of fruit and arthropods in cotton, with implications for binomial sampling. Environ. Entomol. 12:50-54.
35. Xu, X.-M., and Ridout, M. S. 1998. Effects of initial epidemic conditions, sporulation rate, and spore dispersal gradient on the spatio-temporal dynamics of plant disease epidemics. Phytopathology 88:10001012.

36. Xu, X.-M., and Ridout, M. S. 2000. Effects of quadrat size and shape, initial epidemic conditions, and spore dispersal gradient on spatial statistics of plant disease epidemics. Phytopathology 90:738-750.

37. Xu, X.-M., Robinson, J. D., Berrie, A. M., and Harris, D. C. 2001. Spatio-temporal dynamics of brown rot (Monilinia fructigena) on apple and pear. Plant Pathol. 50:569-578. 